\title{
The application of improved genetic algorithms in computer network reliability optimization
}

\author{
Wang LiYan, Zheng XianFeng \\ Department of Computer and Science, College Mobile Telecommunications Chongqing University of \\ Posts and Telecom, China
}

Keywords: improved genetic algorithms; computer network reliability; optimization; cost.

\begin{abstract}
To decrease network link cost is one of the most important pair's for computer network reliability optimization design. In order to make the network cost minimum, the network link medium cost, mathematics model of reliability and resolve algorithm must be considered when it is designed. In the paper, we present an improved genetic algorithm used in optimization calculation for minimizing total link cost and increasing the reliability of the network. Executive procedures of the improved algorithm are described. The simulation results show that improved genetic algorithm provides a method to resolves the network reliability optimization problem which normal approach can not resolve. By using the maneuverable algorithm, optimization process is accelerated, efficiency is increased.
\end{abstract}

\section{Introduction}

The rapid development of computer network technology and its popularity application in various fields, which result in growing increasing of network user node, larger and larger network scales, and increasingly complex of the network. At the same time, people are becoming more and more dependent on computer networks [1]. Once, the failure of the computer network system will cause great loss, even immeasurable. Therefore, the network has become one of the important indicators to measure the overall reliability performance of the computer network system. How to make the network reliability highest, in the condition of reduce the total cost of the network link has became a generally concerned focus of network designers and users are. The traditional method appeared to be inadequate for solving the complex NP optimization problems. In response to this phenomenon, this paper adopted an improved genetic algorithm to calculate the reliability optimization problem of the computer network, at the same time example during the execution of the algorithm was given [2].

\section{Problem description of computer network reliability}

The assumption used by the computer is that there is no more than one straight line to transfer medium connected to the link of between two nodes. So, the computer network can be described by using math graphs $\mathrm{G}=(\mathrm{N}, \mathrm{L})$. And there is not any fault occurs in the network node itself. The reliability of the network link media had no correlation between the lengths of their own. There is only exist two states in the network and network link: the normal work and fault state, while the device failure in the network is random and are independent (including computer network transmission medium and switching equipment). The network link media cost matrix, such as 1 :

$$
C_{0}=\left\{\begin{array}{llll}
C_{11} & C_{12} & \ldots & C_{1 n} \\
C_{21} & C_{22} & \ldots & C_{2 n} \\
\ldots & \ldots & \ldots & \ldots \\
C_{m 1} & C_{m 2} & \ldots & C_{m n}
\end{array}\right\}
$$

$C_{0}:$ Cost matrix; $C_{i j}$ : link media costs between the nodes, among: $1 \leqslant \mathrm{i} \leqslant \mathrm{m}, \quad 1 \leqslant \mathrm{j} \leqslant \mathrm{n}$. The mathematical model of the network link media costs, such as (2), (3) and (4): 


$$
\begin{gathered}
\text { Min } C=\sum_{i=1}^{N} \sum_{j=1}^{i} c_{i j} g_{i j} \\
D_{i} a_{i j} \leq \alpha(i, j=1,2, \ldots, N) \\
\sum_{j=1, j \neq i} g_{i j} \geq \beta(i=1,2, \ldots, N)
\end{gathered}
$$

$\mathrm{C}$ : network costs; $\mathrm{N}$ : the number of nodes; $\mathrm{D}_{\mathrm{i}} \mathrm{a}_{\mathrm{ij}}$ : the number of the shortest logical link media between node $\mathrm{i}$ to $\mathrm{j} ; \alpha, \beta$ : constraints constant of network node reliability. Network link medium reliability matrix such as $(5)$ :

$$
R_{0}=\left\{\begin{array}{llll}
r_{11} & r_{12} & \ldots & r_{1 n} \\
r_{21} & r_{22} & \ldots & r_{2 n} \\
\ldots & \ldots & \ldots & \ldots \\
r_{m 1} & r_{m 2} & \ldots & r_{m n}
\end{array}\right\}
$$

R0: reliability matrix; rij the reliability between link medium nodes, among: $1 \leqslant \mathrm{i} \leqslant \mathrm{m}, \quad 1 \leqslant \mathrm{j} \leqslant \mathrm{n}$.

If the user can communicate with each other in the computer network, then the network is in the state of available. Then at least a spanning tree of the graph $\mathrm{G}$ can be composed by the normal state of the arc and with all the nodes normal [3]. Any time, may be only a subset of L (L') is in the normal state, then the reliability that all the nodes are in normal state is as shown in (6) $\prod_{i}^{N} P\left(n_{i}\right)$

$P\left(n_{i}\right)$ : the reliability of number i link media; $n_{\mathrm{i}}$ : i-node network equipment selection problem abstraction such as (7):

$$
C(x) \leq C
$$

Maximize R(x), while make the formula (7) establish. So, the reliability of mathematical modeling of the entire computer network is as shown below (8):

$$
R(x)=\left\{\sum_{\Omega}\left[\prod_{j \in L^{\prime}} p\left(l_{i}\right)\right] \bullet\left[\prod_{j \in\left(L / L^{\prime}\right)}\left(1-p\left(l_{i}\right)\right)\right]\right\} \bullet \prod_{i}^{N} P\left(n_{i}\right)
$$

$\mathrm{R}(\mathrm{x})$ : the network reliability; $\Omega$ : network used in the collection of a normal state; $\mathrm{L}_{\mathrm{j}}$ : the $\mathrm{jth}$ link medium.

\section{Improved genetic algorithm}

GA'S theory has been extensively presented in several papers in recent years covering a number of applications in power systems. GA is a robust optimization method that works above a set of candidate solutions (individuals) named population and perform a number of operations based on genetic mechanical. Such operators recombine the information contained in the individuals and then, they create new populations.GA uses a selection mechanism which has as its main objective the selecting of "good" individuals from the current population and inserting them into a mating pool. As in the evolutionary theory, GA performs the mechanisms of crossover and mutation, which have the purpose of recombining the genetic material existing in the mating pool, so that better individuals may be created and the search enlarged to other regions of the space. Normally, the average quality of the next population is greater than the previous one[4].Besides the well-known basic GAS operating principles, several modifications and improvements, considered critical to the performance of the optimization process, have been applied with success as a way to make the solution of the different problems feasible. Thus, it is necessary to include the knowledge about the problem into the algorithm. Therefore, a GA developed for a specific problem might fail when used in another problem.

\section{Gene coding}

The first issue that should be defined is the type of representation to be used, so that an individual represents one and only one problem's candidate solution. The most natural representation in the STNEP problem, but not the only one, is assigning one gene for each right-of-way along the power 
system, whether existing or candidate. Thus, the size of the individual corresponds to the sum of the number of existing and candidate rights-of-way[5]. In this paper, a binary one-dimensional encoding is adopted to determine the $\mathrm{N}$ junction point network gene, such as shown in Table 1.

Table.1 Network-node gene

\begin{tabular}{lll}
\hline $\mathrm{N}_{1}$ & $\mathrm{~N}_{2}$ & $\ldots$ \\
\hline $\mathrm{g}_{11}$ & $\mathrm{~g}_{21}$ & $\ldots$ \\
$\ldots$ & $\ldots$ & $\ldots$ \\
$\mathrm{g}_{1 \mathrm{n}}$ & $\ldots$ & $\ldots$ \\
\hline
\end{tabular}

\section{Fitness function selection}

This function is responsible for measuring the quality of the individuals and is completely related to the objective function $\mathrm{f}$. The lower the objective function value evaluated for an individual, the higher its quality. Several fitness functions F may be used, depending on the problem characteristics. The two fitness functions most frequently used are:

and

$$
\mathrm{F}=\mathrm{K}_{1} / \mathrm{f} \text {; usually } \mathrm{K}_{1}=1
$$

$$
\mathrm{F}=\mathrm{K}_{2}-\mathrm{f}
$$

$\mathrm{K} 2$ must be large enough in respect to $\mathrm{f}$ to avoid $\mathrm{F}$ assuming negative values. In this paper, in order to effectively prevent the phenomenon occurrence of deceive of the genetic algorithm, we adopted descending order to the value of the cost of the individual. Individual sort of minimum cost encoding is set to 1, the cost of the largest individual sort encoding to the Pop-size, the fitness function designed is as follow:

$$
f(x)=(x-1) /\left(P_{0} p_{-} \text {size }-1\right)
$$

Where: $\mathrm{x}$ is the position of the individual cost in the sort, Pop-size is the population size and $1 \leqslant \mathrm{x}$ $\leqslant$ Pop-size. Tests performed indicated that there is no significant difference between the improved GA's performance using either one of the two fitness functions.

\section{The selection mechanism}

The selection or sampling mechanism begins the creation of the mating pool by selecting individuals from de current population. In this work, tournament selection is a simple but efficient method of sampling that consists of randomly selecting a predefined number of individuals (tournament or window sizes), and then, pick from this sample the one with the largest fitness value. This process is repeated $\mathrm{N}$ times. Large values of $\mathrm{s}$ increase selective pressure and therefore increase the chance of the GA converging prematurely to a sub-optimal solution. The main advantage of this mechanism is the possibility of controlling the selective pressure, so that the tournament size is a critical parameter for the improved GA's performance. Results obtained using the Tournament Selection mechanism were quite better than those found with the Remainder Stochastic Sampling mechanism because, in the latter, premature convergence was found in almost all cases, due to the appearance of super- individuals in early generations. Tournament selection does not require the implementation of any scaling or ranking method because it only needs the relative differences of the fitness values between the selected individuals.

\section{The crossover mechanism}

In this work, three crossover techniques were implemented and tested: (i) at one-point, (ii) at two-points and (iii) "by mask". The crossover at two-point crossover has been referenced as a fairly suitable crossover technique, which led us to select this technique. However, we could not observe its superior performance with regard to the others.

\section{The mutation mechanism}

From the viewpoint of genetic diversity, this mechanism is especially important to prevent the permanent loss of any allele. After several generations, it could be possible for a given gene to have the same allele in all the individuals. The mutation mechanism might restore missed alleles, leading the search to regions possibly not yet explored, acting as a source of diversity. By this mechanism, for each gene, an uniform random number is generated into the interval $[0,1]$. If this number is lower than the mutation rate, the respective allele of the current gene is swapped for another random value within a specific interval. The adopted intervals and the way of selecting the new alleles depend on the 
specific case of study and are subsequently detailed. In our work, an increasing mutation rate was implemented in order to enhance the local search around the optimum solution. Nevertheless, the use of such a procedure would be inefficient for the STNEP problem if the classical mechanism for performing the mutation was adopted. Typically, in the classical mechanism, an allele can assume any value within the feasible interval. Thus, to overcome this problem, we implemented a procedure based on a Simulated Annealing Approach in order to control the mutation procedure, which is presented in the following. First, need to confirm the mutated gene and the number; second, selected in accordance with the selected range after the new gene fragment to replace the old gene fragments in order to generate offspring. Usually mutation rate within the range of from 0.001 to 0.01 , but sometimes if the mutation genes that produce the wrong network connectivity structure, need to be adjusted operation.

\section{Simulation example}

The below shown is the network reliability optimization example. Formula (12) and (13) are respectively stand the medium cost and reliability of the network link matrix.

$$
C_{0}=\left(\begin{array}{cccccc}
0 & 4 & 6 & 13 & 16 & 6 \\
4 & 0 & 8 & 2 & 6 & 11 \\
6 & 8 & 0 & 4 & 10 & 8 \\
13 & 2 & 4 & 0 & 1 & 6 \\
16 & 6 & 10 & 1 & 0 & 5 \\
6 & 11 & 8 & 6 & 5 & 0
\end{array}\right) \quad R_{0}=\left(\begin{array}{cccccc}
0 & 0.968 & 0.947 & 0.991 & 0.999 & 0.960 \\
0.968 & 0 & 0.972 & 0.987 & 0.996 & 0.990 \\
0.947 & 0.972 & 0 & 0.960 & 0.947 & 0.997 \\
0.991 & 0.987 & 0.960 & 0 & 0.925 & 0.921 \\
0.999 & 0.996 & 0.947 & 0.925 & 0 & 0.972 \\
0.960 & 0.990 & 0.997 & 0.921 & 0.972 & 0
\end{array}\right)
$$

The network nodes $\mathrm{N}=6$. The network node reliability constraints constants $\alpha=2, \beta=2$. The number of iteration that the genetic manipulation is 200 times during simulation solving. The total cost of the network link media is 45 , and the maximum that ensure network reliability is 0.78 .

\section{Conclusion}

This article adopted improved genetic algorithm applied to computer network reliability optimization calculation. The purposes are to reduce the network link media costs, and improve network reliability purpose. The algorithm is simple, programming is easy to implement and readable. Simulation demonstrated the practical value of the improved genetic algorithm for solving the above problems.

\section{References}

[1]Zhang Y, Liu Y Q. Soft calculation method.Beijing: Science Press,2002.

[2]Wang DW, Tang J F, Huang M. Genetic algorithms and engineering design Beijing: Science Press, 2000.

[3]Wei L Y,Liu Z Q. The computer network inter locks the equipment Beijing: Greatly Permanent Electronic Press, 1999.

[4] Szczerbicka H, Becker M, Syrjakow M. Genetic algorithms: A tool for modeling, simulation and optimization of complex systems. Cybernetics and Systems, 1998, 29(7):639- 659.

[5] Altiparmak F, Dengiz B, Smith A E. Reliability optimization of computer communication networks using genetical gorithms. Dawid S, Fanshye. SMC'98 Conference Proceedings. IEEE, 1998: $4676-4681$. 